\title{
Curie temperature, exchange integrals, and magneto-optical properties in off-stoichiometric bismuth iron garnet epitaxial films
}

\author{
B. Vertruyen, ${ }^{1, *}$ R. Cloots, ${ }^{1}$ J. S. Abell, ${ }^{2}$ T. J. Jackson, ${ }^{2}$ R. C. da Silva, ${ }^{3}$ E. Popova, ${ }^{4}$ and N. Keller ${ }^{4}$ \\ ${ }^{1}$ SUPRATECS/LCIS, Chemistry Institute B6, University of Liege, B-4000 Liege, Belgium \\ ${ }^{2}$ School of Engineering, The University of Birmingham, B15 2TT Edgbaston, Birmingham, United Kingdom \\ ${ }^{3}$ Laboratório de Feixes de Iões, Departamento Física, Instituto Tecnologico e Nuclear, Sacavém, Portugal \\ ${ }^{4}$ Groupe d'Etude de la Matière Condensée (GEMaC), CNRS-UVSQ, 45 Avenue des Etats-Unis, 78035 Versailles Cedex, France
}

(Received 22 November 2007; revised manuscript received 25 June 2008; published 30 September 2008)

\begin{abstract}
We have studied the influence of the stoichiometry on the structural, magnetic, and magneto-optical properties of bismuth iron garnet $\left(\mathrm{Bi}_{3} \mathrm{Fe}_{5} \mathrm{O}_{12}\right)$ thin films grown by pulsed laser deposition. Films with different stoichiometries have been obtained by varying the $\mathrm{Bi} / \mathrm{Fe}$ ratio of the target and the oxygen pressure during deposition. Stoichiometry variations influence the Curie temperature $T_{C}$ by tuning the (Fe)-O-[Fe] geometry: $T_{C}$ increases when the lattice parameter decreases, contrary to what happens in the case of stoichiometric rare-earth iron garnets. The thermal variation of the magnetization, the Faraday rotation, and the Faraday ellipticity have been analyzed in the frame of the Néel two-sublattice magnetization model giving energies of $-48 \mathrm{~K}(4.1 \mathrm{meV}),-29 \mathrm{~K}(2.5 \mathrm{meV})$, and $84 \mathrm{~K}(7.3 \mathrm{meV})$ for the three magnetic exchange integrals $j_{a a}, j_{d d}$, and $j_{a d}$, respectively. Magneto-optical spectroscopy linked to compositional analysis by Rutherford backscattering spectroscopy shows that $\mathrm{Bi}$ and/or Fe deficiencies also affect the spectral variation (between 1.77 and $3.1 \mathrm{eV}$ ). Our results suggest that bismuth deficiency has an effect on the magneto-optical response of the tetrahedral Fe sublattice, whereas small iron deficiencies affect predominantly the magneto-optical response of the octahedral sublattice.
\end{abstract}

DOI: 10.1103/PhysRevB.78.094429

PACS number(s): 75.50.Gg, 78.20.Ls, 75.30.Et, 61.50.Nw

\section{INTRODUCTION}

Bismuth substitution on the dodecahedral site of ferrimagnetic iron garnets is known to enhance drastically the Faraday rotation in visible light (Ref. 1 and references therein). Apart from the obvious interest of these Bi-substituted compounds in view of applications, ${ }^{2-9}$ there has also been much attention paid to the theoretical aspects (see Ref. 10) especially concerning the influence of $\mathrm{Bi}$ on the spectral dependence of the magneto-optical properties. ${ }^{11-19}$ However, studies on the role of bismuth in iron garnets have long been confined to compounds with relatively low bismuth content because the pure bismuth iron garnet $\mathrm{Bi}_{3} \mathrm{Fe}_{5} \mathrm{O}_{12}$ (BIG) does not exist in bulk form (due to the large ionic radius of the $\mathrm{Bi}^{3+}$ cation) and cannot be grown by liquid-phase epitaxy (LPE). Since 1989, several authors have shown that BIG can be grown as a thin film on suitable garnet substrates by pulsed laser deposition (PLD), 4,6,18,20-28 reactive ion-beam sputtering (RIBS) (Refs. 29-31), or other vapor deposition techniques. ${ }^{32-34}$ Much information has been gained through these works but comparison of the available data for BIG films reveals a significant dispersion of the experimental results. For example, Curie temperatures vary between 618 and $643 \mathrm{~K}$ (Refs. 21, 31, 35, and 36) and typical values of the saturation magnetization $\mu_{0} M_{s}$ range from 0.12 to 0.165 T. ${ }^{20,22-24,30,31}$ The cubic lattice parameter is certainly an influent parameter since, depending on the garnet substrate, the film lattice-parameter values vary between 1.2619 and $1.2671 \mathrm{~nm} .{ }^{22,23,28,30-33,37}$ However, another important issue is the stoichiometry of the films: contrary to liquid phase epitaxy, vapor phase deposition techniques, such as used to grow BIG films frequently yield off-stoichiometric films, ${ }^{38}$ and the reported $\mathrm{Bi} / \mathrm{Fe}$ ratios vary indeed in a wide range (from 0.48 to 0.66$)$. $2,26,28,30,33,34,39$
In the present paper we study the effect of variations to the stoichiometry of bismuth iron garnet thin films on the magnetic and magneto-optical properties. We report on the temperature dependence (100-700 K) of the Faraday rotation and ellipticity at a photon energy of $2.25 \mathrm{eV}$ for BIG films grown by PLD under different oxygen pressures and from targets of different compositions. These magneto-optical properties give us access to the magnetization and the Curie temperature. The magnetic sublattice exchange integrals are obtained by a self-consistent calculation and fitting of the Néel two-sublattice model to the experimental data. The relations of the Curie temperature and exchange integrals with the lattice parameter and the stoichiometry of the films are discussed in Sec. III B.

These parameters also appear to affect the spectral dependence (1.77 to $3.1 \mathrm{eV}$ ) of the Faraday rotation and ellipticity at room temperature. In the literature, the quantitative analysis of the influence of bismuth substitution on the Faraday rotation spectra has attracted much interest ${ }^{11-19}$ but was mainly investigated on stoichiometric films grown by LPE, i.e., with relatively low bismuth content. Building on ideas from Dionne and Allen, ${ }^{11,12}$ Helseth et al. ${ }^{13,14}$ analyzed the Faraday rotation spectra with a simple model based on two optical transitions. Recently, Dionne and Allen ${ }^{15}$ have discussed the concept of intersublattice transitions and proposed a crystalline electric level scheme based on molecular-orbital hybrid states. This analysis allowed them to assign the observed transition energies in the experimental dielectric functions to excitations occurring on the tetrahedral and octahedral sites of Fe-O polyhedra. Another approach proposed by Zenkov and Moskvin ${ }^{16,17}$ based on a semiquantitative model calculating explicitly the charge-transfer transitions in the octahedral $\left(\mathrm{FeO}_{6}\right)^{9-}$ and tetrahedral $\left(\mathrm{FeO}_{4}\right)^{5-}$ complexes 
TABLE I. Nominal $\mathrm{Bi} / \mathrm{Fe}$ ratio in target, $P\left(\mathrm{O}_{2}\right)$ during deposition and cooling, film thickness measured by $\mathrm{RBS}$, Bi/Fe ratio in film, Bi deficiency $\left\{\delta_{\mathrm{Bi}}=\left(3-x_{\mathrm{Bi}}\right) / 3\right\}$, and Fe deficiency $\left\{\delta_{\mathrm{Fe}}=\left(5-x_{\mathrm{Fe}}\right) / 5\right\}$ measured by RBS, crystallographic cell parameter $a$, Curie temperature $T_{C}$, room-temperature saturation magnetization $\mu_{0} M_{s}$, and coercive field $\mu_{0} H_{C}$

\begin{tabular}{lcccccccccc}
\hline \hline Film & Bi/Fe target & $P_{\mathrm{O} 2}(\mathrm{mTorr})$ & $d(\mathrm{~nm})$ & $\mathrm{Bi} / \mathrm{Fe}(\mathrm{RBS})$ & $\delta_{\mathrm{Bi}}(\mathrm{RBS})$ & $\delta_{\mathrm{Fe}}(\mathrm{RBS})$ & $(\mathrm{nm})$ & $T_{C}(\mathrm{~K})$ & $\begin{array}{c}\mu_{0} M_{S} \\
(\mathrm{~T})\end{array}$ & $\mu_{0} H_{C}(\mathrm{mT})$ \\
\hline$A$ & $3 / 5$ & 500 & 805 & 0.50 & 0.20 & 0.03 & $1.2621 \pm 0.0005$ & 701 & $0.113 \pm 0.024$ & 6.5 \\
$B$ & $3 / 5$ & 250 & 430 & 0.49 & 0.25 & 0.08 & $1.2616 \pm 0.0005$ & 695 & $0.127 \pm 0.018$ & 6.9 \\
$C$ & $3 / 5$ & 50 & 690 & 0.50 & 0.20 & 0.03 & $1.2638 \pm 0.0005$ & 687 & $0.133 \pm 0.023$ & 7.3 \\
$D$ & $3.15 / 5$ & 50 & 400 & $\leq 0.59$ & $\geq 0.06$ & 0.04 & $1.2639 \pm 0.0005$ & 675 & $0.142 \pm 0.020$ & 4.5 \\
$E$ & $3.3 / 5$ & 50 & 400 & 0.61 & 0.04 & 0.05 & $1.2653 \pm 0.0005$ & 653 & $0.140 \pm 0.019$ & 3.6 \\
\hline \hline
\end{tabular}

gives a quantitative basis to the semiempirical assignment of the observed transition energies to excitations occurring in different $\mathrm{Fe}-\mathrm{O}$ coordinations. Concerning the description of the electronic structure, these two approaches used to be the only means to assign the observed transitions to excitations in the Fe-O polyhedra. Only very recently, the first fully relativistic band-structure calculation performed by Oikawa et $a l .{ }^{19}$ reporting a density-functional theory calculation of the electronic structure of $\mathrm{Bi}_{3} \mathrm{Fe}_{5} \mathrm{O}_{12}$ became available. Concerning completely substituted (and possibly nonstoichiometric) $\mathrm{Bi}_{3} \mathrm{Fe}_{5} \mathrm{O}_{12}$, studies giving an interpretation of the observed optical and magneto-optical transition lines within the frame of the above sketched theoretical interpretations are scarce: Kahl et al. ${ }^{18}$ found that a single diamagnetic transition seems to be sufficient to describe the Faraday rotation spectrum between 1.24 and $2.4 \mathrm{eV}$. In Sec. III C, we use the simple two-transition model based on the works by Zenkov et al., ${ }^{16,17}$ Dionne et al., ${ }^{11,12,15}$ and Helseth et al. ${ }^{13,14}$ to suggest a qualitative link between the modified spectral response and a possible site preference for the cationic vacancies.

\section{EXPERIMENTAL METHODS}

BIG films were deposited on $5 \times 5 \mathrm{~mm}^{2} \quad(001)$ $\mathrm{Gd}_{3} \mathrm{Ga}_{5} \mathrm{O}_{12}$ (GGG) substrates by laser ablation with a $\mathrm{KrF}$ excimer laser (Lambda Physik LPX210, $\lambda=248 \mathrm{~nm}$, pulse energy density $\sim 2.5 \mathrm{~J} / \mathrm{cm}^{2}$, and a repetition rate of $50 \mathrm{~Hz}$ ). Targets with different compositions were used. The first was a commercial target with $3 / 5$ nominal $\mathrm{Bi} / \mathrm{Fe}$ ratio. The other two were prepared by standard solid state reaction of $\mathrm{Bi}_{2} \mathrm{O}_{3}$ and $\mathrm{FeC}_{2} \mathrm{O}_{4} \cdot 2 \mathrm{H}_{2} \mathrm{O}$ with nominal $\mathrm{Bi} / \mathrm{Fe}$ ratios of $3.15 / 5$ and $3.3 / 5$. The distance between the substrate and the target was $5.5 \mathrm{~cm}$. The temperature of the substrate holder was kept at $570{ }^{\circ} \mathrm{C}$ during deposition. The films were cooled at $10{ }^{\circ} \mathrm{C} / \mathrm{min}$ immediately after ablation. The deposition parameters for each film can be found in Table I.

General x-ray diffraction characterization was performed with a four-circle Siemens D5000 diffractometer $\left(\mathrm{Cu} K_{\alpha}\right.$ radiation). $\mathrm{Cu} K_{\alpha 1} \theta / 2 \theta$ scans and rocking curves were collected with a PANalytical X'Pert PRO MRD for precise determination of the cell parameters.

The composition of the films was studied by Rutherford backscattering spectroscopy (RBS). The films were irradiated with $2 \mathrm{MeV}{ }^{4} \mathrm{He}^{+}$ions with beam currents of 1-3 nA and total accumulated charges of $1-2 \mu \mathrm{C}$. All spectra were normalized to $1 \mu \mathrm{C}$ charge. The RBS spectra were collected with two semiconductor $\mathrm{Si}$ detectors, placed at angles of $140^{\circ}$ and slightly less than $180^{\circ}$ with respect to the incoming beam direction, and having energy resolutions of 13 and 16 $\mathrm{keV}$, respectively. For each film, two sets of spectra were taken sequentially in the same conditions in order to make sure that the analyzing beam did not induce any change in the samples due to ion irradiation. In all cases the spectra overlapped entirely. The spectra were obtained under $3^{\circ}$ tilt in order to avoid channeling.

Temperature-dependent Faraday rotation measurements have been performed using a custom-designed magnetooptical magnetometer. ${ }^{40}$ The samples were mounted successively in a high-temperature furnace and in a liquid nitrogen cryostat allowing variation of the sample temperature from 100 to $900 \mathrm{~K}$. Measurements were performed at fixed photon energies of 1.77, 1.90, 2.06, 2.25, 2.48, 2.76, 3.10 eV and in spectroscopic mode with a second setup based on a phase modulation technique using a commercial charge coupled device (CCD) spectrometer.

\section{RESULTS AND DISCUSSION}

\section{A. Structure and composition of the films}

The homogeneity and epitaxy of all films were characterized by x-ray diffraction. Each $\theta / 2 \theta$ pattern displays the $(00 \ell)$ peaks of the GGG substrate and the BIG garnet film as shown in Fig. 1 for film $\mathrm{C}$. This confirms that the garnet phase is obtained for a rather large range of experimental conditions as reported by most other authors. ${ }^{26,30}$ Tepper et $a l .{ }^{28}$ reported a narrower window of deposition conditions, maybe due to the fact that they used a low laser energy density for the deposition $\left(1.4 \mathrm{~J} / \mathrm{cm}^{2}\right)$. Traces of $\mathrm{BiFeO}_{3}$ are observed in films $A, C$, and $E$. Slightly more intense $\mathrm{BiFeO}_{3}$ peaks are found in film $D$. Adachi et al. $^{22}$ also detected a weak $\mathrm{BiFeO}_{3}$ peak in films grown by PLD with oxygen pressure above 30 mTorr. Epitaxial cube-on-cube growth is confirmed by $\Phi$ scans of the (4010) reflection (see inset of Fig. 1). The typical full width at half maximum (FWHM) of the (008) BIG reflection is in the range $0.20^{\circ}-0.25^{\circ}$ similar to other published work for growth on GGG substrates. ${ }^{24,32}$ By using substrates with a better lattice match, such as GSGG, ${ }^{22}$ the FWHM can be reduced to $0.06^{\circ}$. The lattice parameters determined for our films are reported in Table I. They were obtained by applying the Nelson-Riley extrapolation. ${ }^{22,41}$ 


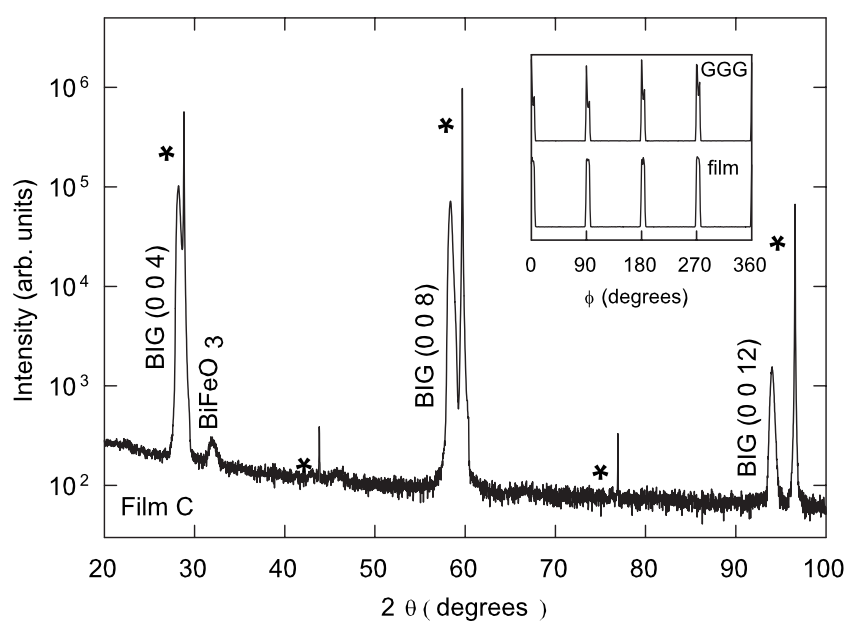

FIG. 1. X-ray diffraction results. Main figure: $K_{\alpha 1} \theta / 2 \theta$ scan of film $C$ in logarithmic scale. GGG reflections are marked by an asterisk. Inset: $\Phi$ scan of the (4010) reflection.

Lattice parameters published for BIG in the literature vary between 1.2620 and $1.2671 \mathrm{~nm}$ with an average value of $a$ $=1.2632 \pm 0.0015 \mathrm{~nm} .{ }^{22,23,28,30-33,37}$ More specifically, for BIG growth on GGG substrate, the observed lattice parameters vary in the range of 1.2619 to $1.2635 \mathrm{~nm} .{ }^{28,32}$ In the present work, the lattice parameters vary between 1.2616 and $1.2653 \mathrm{~nm}$ depending on the oxygen pressure and target composition.

The composition of the films was studied by RBS. RBS channeling experiments were performed in order to discard the influence of channeling on the experimental data and confirmed the rather good epitaxial quality of the films. For film $C$, the channeling quality (given by the ratio of RBS yields in aligned and off-aligned situations) is about 22\% (to be compared to less than $4 \%$ for the single-crystalline GGG substrate). Figure 2 shows the RBS experimental data and the simulated spectrum in the case of film $E$. It was not possible to reproduce the fine details of the RBS spectra with a constant composition description. For all films, the results suggest an overall decrease of the $\mathrm{Bi} / \mathrm{Fe}$ ratio from the BIG/GGG interface to the BIG surface as also observed

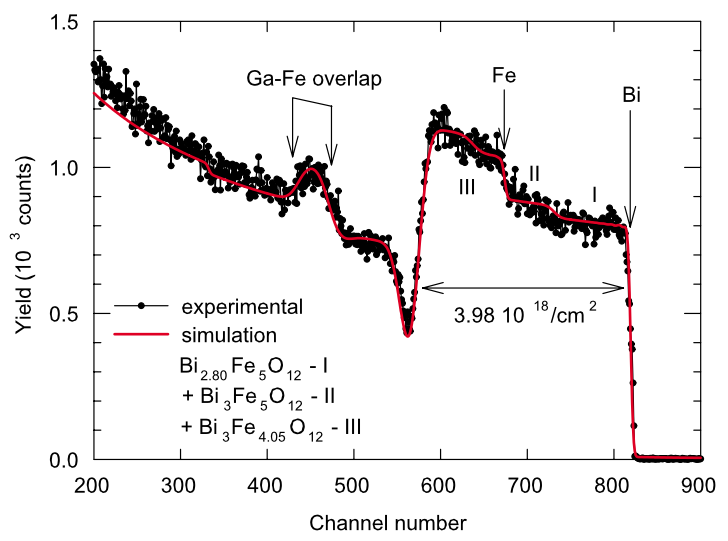

FIG. 2. (Color online) RBS spectrum of film $E$. The experimental data are fitted by a model with three layers of decreasing $\mathrm{Bi} / \mathrm{Fe}$ ratio from the BIG/GGG interface to the BIG surface.

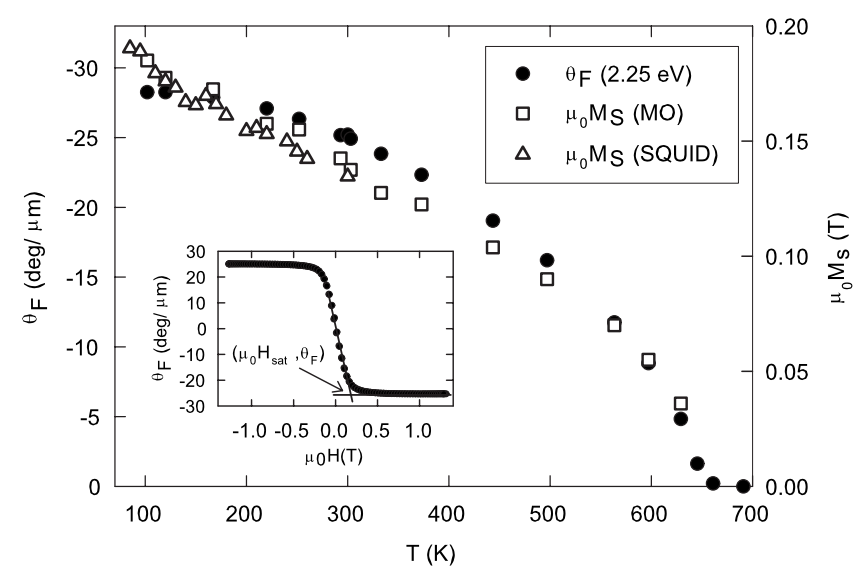

FIG. 3. Temperature dependence of the Faraday rotation measured at $E=2.25 \mathrm{eV}$, of the saturation magnetization, and of the saturation magnetization measured by SQUID for film $E$. The inset shows a typical hysteresis loop $\theta_{F}(H)$ indicating how the values of Faraday rotation and magnetization are determined. $H_{\text {sat }}$ is the saturation field (see text for details).

by other authors for BIG films deposited by PLD. ${ }^{28,39}$ Previously, Krumme et al. ${ }^{42}$ had concluded from their experiments on Bi-substituted iron garnet films grown by rf magnetron sputtering that the $\mathrm{Bi}-\mathrm{O}$ bond is weaker than other metaloxygen bonds in iron garnets. The average values for $\mathrm{Bi} / \mathrm{Fe}$ ratio, Bi deficiency $\left\{\delta_{\mathrm{Bi}}=\left(3-x_{\mathrm{Bi}}\right) / 3\right\}$, and Fe deficiency $\left\{\delta_{\mathrm{Fe}}\right.$ $\left.=\left(5-x_{\mathrm{Fe}}\right) / 5\right\}$ can be found in Table I. Since some $\mathrm{BiFeO}_{3}$ was detected by $\mathrm{x}$-ray diffraction, the $\mathrm{Bi} / \mathrm{Fe}$ ratio in the garnet phase could be somewhat overestimated especially for film $D$. These RBS stoichiometry data suggest that films deposited from the stoichiometric target (films $A, B$, and $C$ ) are strongly Bi-deficient as also observed by other authors. ${ }^{26,39}$ Films $D$ and $E$ were grown from targets containing a bismuth excess and have a $\mathrm{Bi} / \mathrm{Fe}$ ratio close to the nominal value of 0.6 .

The variations of crystallographic parameter and composition will be discussed later in this paper in relation with the magneto(-optical) properties.

\section{B. Temperature dependence of the magnetization and the Faraday rotation}

The temperature dependence of the saturation magnetization $M_{S}$ can be determined using magneto-optical Faraday rotation measurements. ${ }^{38}$ Using the anisotropy constants reported by Adachi et al. ${ }^{22}$ for BIG films, it can be shown that the magnetocrystalline anisotropy field $H_{K}$ is approximately one order of magnitude smaller than the demagnetizing field $H_{d}$ (with $H_{d} \approx M_{S}$ when $H$ is perpendicular to the film plane). Therefore, the extrapolated magnetic saturation field $\left\{\mu_{0} H_{\text {sat }}=\mu_{0}\left(H_{d}+H_{K}\right)\right\}$ indicated by the arrow in the inset of Fig. 3 can be used to determine the saturation magnetization $M_{S}$ when neglecting $H_{K}$ in the first approximation. The room-temperature magnetization values are given in Table I. A maximum value of $\mu_{0} M_{S} \approx 0.14 \mathrm{~T}$ is obtained for the stoichiometric BIG films (films $D$ and $E$ ). Typical values reported in the literature are in the range $0.12-0.165 \mathrm{~T}$ for films grown by PLD or RIBS on various substrates..$^{20,22-24,30,31}$ 


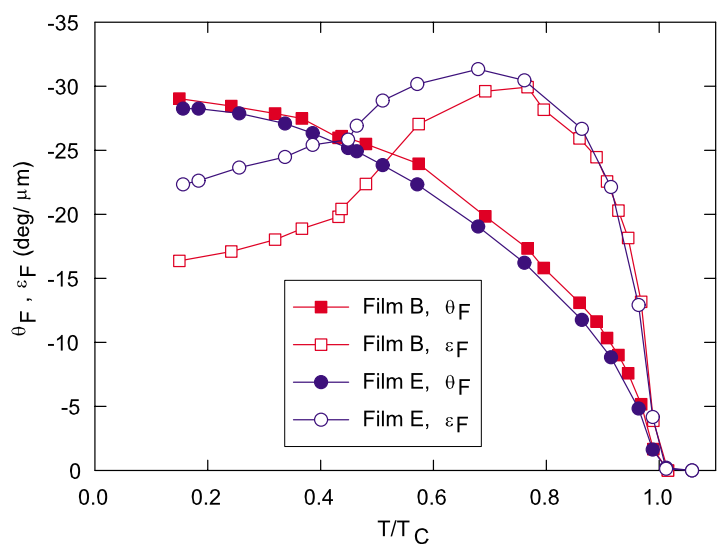

FIG. 4. (Color online) Temperature dependence of the Faraday rotation $\left(\theta_{F}\right)$ and ellipticity $\left(\varepsilon_{F}\right)$ at $E=2.25 \mathrm{eV}$ as a function of reduced temperature $T / T_{C}$ for films $B$ and $E$. Plain lines are guides for the eye.

Films $D$ and $E$ display the lowest coercive fields of the series $(\sim 4 \mathrm{mT})$, which is two and four times smaller than the coercive fields determined by Kahl et al. ${ }^{24}$ and Okuda et al., ${ }^{33}$ respectively.

Figure 3 shows the typical temperature dependence of the Faraday rotation (full dots) as measured on film $E$ for a photon energy of $2.25 \mathrm{eV}$ and in the temperature range of 100 to $700 \mathrm{~K}$. The same figure compares the saturation magnetization values deduced from the Faraday rotation data as described above (square symbols) with the values measured with a superconducting quantum interference device (SQUID) magnetometer (open triangles). The SQUID data agree well with the magnetization data obtained from the Faraday hysteresis loops confirming that $H_{K}$ is small compared to $M_{S}$. The temperature dependence of the Faraday rotation of BIG displays a regular decrease of $\theta_{F}$ with increasing temperature, contrary to the case of yttrium iron garnet (YIG), ${ }^{38,43-46}$ for which the presence of two magnetic sublattices is evidenced by a maximum at $\sim 350 \mathrm{~K}$. This trend on $\mathrm{Bi}$ substitution had already been observed by Hansen et $a l .{ }^{44}$ for $\mathrm{Y}_{3-x} \mathrm{Bi}_{x} \mathrm{Fe}_{5} \mathrm{O}_{12}(x \leq 1.44)$ compounds. Although the temperature dependence of the Faraday rotation of BIG might look as if only one magnetic sublattice were present, the normalized temperature dependence $\left(T / T_{C}\right)$ of the ellipticity displayed in Fig. 4 clearly confirms the presence of the two magnetic sublattices (tetrahedral and octahedral Fe-O polyhedra) via a pronounced maximum. As in the case of YIG (see Ref. 38), the observed maximum here in the Faraday ellipticity does shift with changes to stoichiometry and could be an indicator of the relative occupation of octahedral and tetrahedral iron sites. The magneto-optical spectroscopy data discussed in Sec. III C seem to indicate that film $B$ presents an increased iron deficiency of the octahedral $\mathrm{Fe}-\mathrm{O}$ polyhedron leading to an increase in the reduced temperature of the maximum in Faraday ellipticity as has been observed for $\theta_{F}(T)$ in $\mathrm{YIG}^{38}$

Another important property of a magnetic material is the Curie temperature as it is directly related to the exchange integrals. All determined Curie temperatures can be found in Table I. These values ranging between 653 and $701 \mathrm{~K}$ are

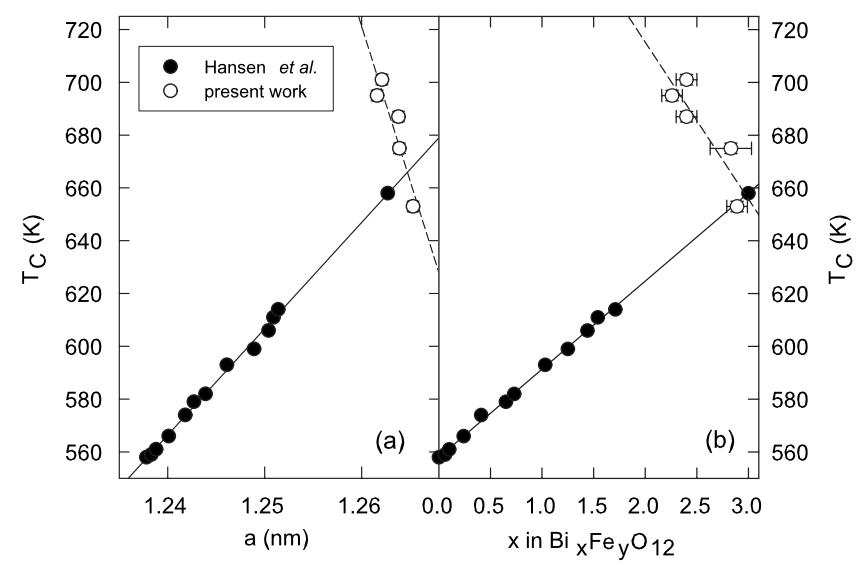

FIG. 5. Curie temperature as a function of (a) cubic cell parameter and (b) Bi content in the film. Black symbols are data taken from Hansen et al. (Refs. 44-46) for (Y,Bi)IG films. The point at $1.263 \mathrm{~nm} ; x_{\mathrm{Bi}}=3$ results from a linear extrapolation of these data. Empty symbols are the data from the present work. The dashed line is a linear fit to these data.

higher than the experimental values reported in the literature, ${ }^{21,31,35,36}$ which do not exceed $643 \mathrm{~K} .{ }^{31}$ The Curie temperatures for the Bi-deficient films are even higher than the value of $672 \mathrm{~K}$ extrapolated from the data of Hansen et $a l .{ }^{45}$ for Bi-substituted YIG. Figures 5(a) and 5(b) give a graphical representation of the measured Curie temperatures as a function of the lattice parameter [Fig. 5(a)] and of the bismuth content [Fig. 5(b)] compared with data taken from literature (solid circles) on bismuth substituted YIG films grown by LPE. ${ }^{44-46}$ It can be seen that nonstoichiometry affects strongly the Curie temperature. Compared to $\mathrm{Bi}$ substituted YIG films, ${ }^{44}$ Bi-deficient BIG films display inverse dependencies of $T_{C}$ on lattice parameter $a$ and bismuth concentration $x_{\mathrm{Bi}}$, i.e., $T_{C}$ tends to increase when $a$ or $x_{\mathrm{Bi}}$ decreases.

\section{Analysis of magnetization data}

The thermal variation of the magnetization, the Faraday rotation, and the Faraday ellipticity can be described in the frame of Néel model as the result of two antiferromagnetic (AF) coupled sublattices. Formally, the magnetization of $\mathrm{Bi}_{3} \mathrm{Fe}_{5} \mathrm{O}_{12}$ is given by (cf. Ref 47)

$$
M(T)=M_{d}(T)-M_{a}(T),
$$

where $M_{d}$ and $M_{a}$ are the magnetization of the tetrahedral and octahedral sublattices, respectively.

Each sublattice magnetization is given by

$$
M_{i}(T)=M_{i}(0) B_{s i}\left(x_{i}\right),
$$

where $B_{s i}\left(x_{i}\right)$ represents the Brillouin function of the $i$ th sublattice $(i=a, d)$,

$$
B_{s i}=\frac{2 S_{i}+1}{2 S_{i}} \operatorname{coth}\left[\left(\frac{2 S_{i}+1}{2 S_{i}}\right) x_{i}\right]-\frac{1}{2 S_{i}} \operatorname{coth}\left[\left(\frac{1}{2 S_{i}}\right) x_{i}\right],
$$

and 


$$
M_{d}(0)=3 g S_{d} \mu_{B} N_{A} f_{d}, \quad M_{a}(0)=3 g S_{a} \mu_{B} N_{A} f_{a} .
$$

Here the constants $f_{a}$ and $f_{d}$ have been introduced to allow for a variation of the zero-temperature magnetization in each sublattice. $S_{k}$ represents the spin quantum numbers of the magnetic ions with $S_{k}=5 / 2$ for $\mathrm{Fe}^{3+}$ in the high spin state. $\mu_{B}$ is the Bohr magneton $\left(=9.2741 \times 10^{-24} \mathrm{~J} / \mathrm{T}\right), k_{B}$ is the Boltzmann constant $\left(=1.3807 \times 10^{-23} \mathrm{~J} / \mathrm{K}\right)$, and $N_{A}$ is the Avogadro constant $\left(=6.022 \times 10^{23} \mathrm{~mol}^{-1}\right)$.

The coefficients $x_{d}$ and $x_{a}$ are given by

$x_{d}=\frac{S_{d} g \mu_{B}}{k_{B} T}\left(N_{d d} M_{d}+N_{d a} M_{a}\right), \quad x_{a}=\frac{S_{a} g \mu_{B}}{k_{B} T}\left(N_{a a} M_{a}+N_{a d} M_{d}\right)$

and $N_{i k}=\left(2 Z_{i k} J_{i k}\right) /\left(n_{k} g_{i} g_{k} \mu_{B}^{2}\right)$, where $Z_{i k}$ is the number of nearest neighbors, $n_{k}$ is the number of ions per mole in the $k$ th sublattice, $J_{i k}$ is the exchange integral $\left(J_{a d}=J_{d a}\right)$, and $g_{l}$ is the spectroscopic splitting factor of each sublattice $(=2.0)$. Equation (1) can be self-consistently solved in order to obtain the exchange integrals $J_{i k}$, which govern the magnetic order and which determine the Curie temperature of the system. In order to perform suitable numerical adjustment of Eq. (1) to the experimental data, it is preferable to normalize Eqs. (1)-(5) in order to express them in units of $\mu_{B}$. The magnetization scales as $n_{B}=M_{S} / \mu_{B} N_{A}$. Normalizing Eqs. (1)-(5), one obtains for the reduced magnetization,

$$
n_{B}(T)=3 g S_{d} f_{d} B_{s d}\left(x_{d}^{*}\right)-2 g S_{a} f_{a} B_{s a}\left(x_{a}^{*}\right)
$$

with

$$
\begin{gathered}
x_{d}^{*}=\frac{2 S_{d}}{g T}\left(j_{d d} n_{d}+j_{d a} n_{a}\right), \quad x_{a}^{*}=\frac{2 S_{a}}{g T}\left(j_{a a} n_{a}+j_{a d} n_{d}\right), \\
j_{k l}=J_{k l} / k_{B},
\end{gathered}
$$

where $j_{k l}$ is expressed in kelvins. Now the magneto-optical Faraday rotation and ellipticity are both proportional to a combination of the individual sublattice magnetizations weighted by a proportionality factor. One may write them as follows:

$$
\begin{aligned}
& \theta_{F}(T)=C_{V, d} 3 g S_{d} f_{d} B_{s d}\left(x_{d}^{*}\right)-C_{V, a} 2 g S_{a} f_{a} B_{s a}\left(x_{a}^{*}\right), \\
& \varepsilon_{F}(T)=C_{E, d} 3 g S_{d} f_{d} B_{s d}\left(x_{d}^{*}\right)-C_{E, a} 2 g S_{a} f_{a} B_{s a}\left(x_{a}^{*}\right) .
\end{aligned}
$$

Equations (6) and (8) were integrated into a nonlinear leastmean-square fitting algorithm using a self-consistent determination of each sublattice magnetization for the adjustable parameters $\left(j_{i k}, f_{i}, C_{v, I}\right.$, and $C_{E, I}$ with $\left.i=a, d\right)$. The algorithm allows for a simultaneous adjustment of Eqs. (6) and (8) to the respective data sets. The obtained fitting for film $A$ is given in Fig. 6 where the magnetization, the Faraday rotation, and the Faraday ellipticity have been adjusted. The figure shows the calculated thermal variations for $M_{S}, \theta_{F}$, and $\varepsilon_{F}$ and the two sublattice magnetizations of the tetrahedral and octahedral sublattice (dashed lines). Figure 7 gives the results of this analysis for the exchange integrals $j_{a a}, j_{d d}$, and $j_{a d}$ as a function of the lattice parameter [Fig. 7(a)] and as a function of both the bismuth deficit, $\delta_{\mathrm{Bi}}$ [Fig. 7(b)], and the iron deficit, $\delta_{\mathrm{Fe}}[$ Fig. $7(\mathrm{c})]$. The experimentally determined

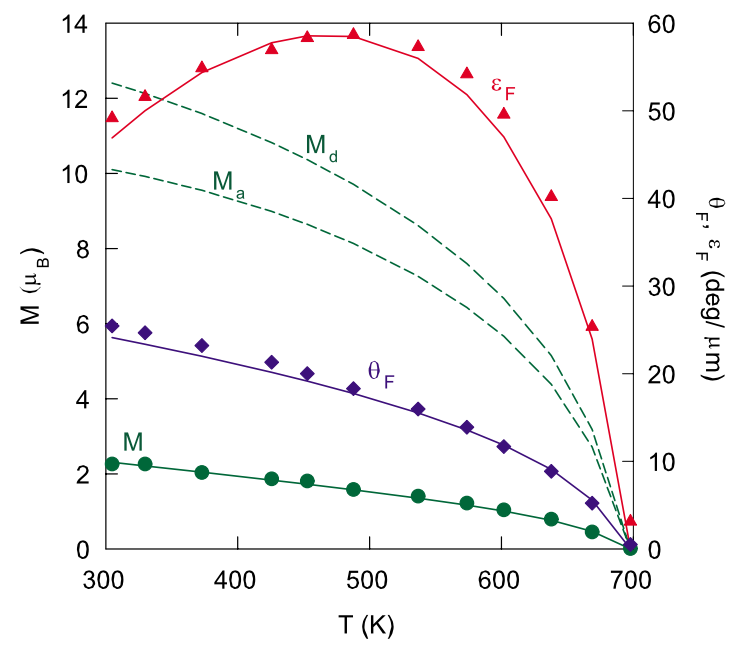

FIG. 6. (Color online) Temperature dependence of the magnetization (circles), Faraday rotation (diamonds) and ellipticity (triangles) for film $A$ shown with the corresponding numerical fits (plain lines; see text for details), and with the calculated sublattice magnetization for the octahedral $\left(M_{a}\right)$ and tetrahedral $\left(M_{d}\right)$ sublattices (dashed lines).

Curie temperatures are also displayed on the same figures. Both $j_{a a}$ and $j_{d d}$ have negative values characteristic of ferromagnetic interactions, whereas $j_{a d}$ is positive revealing the expected antiferromagnetic intersublattice coupling. For our BIG films, typical values of $-48 \mathrm{~K}(4.1 \mathrm{meV}),-29 \mathrm{~K}(2.5$ $\mathrm{meV})$, and $84 \mathrm{~K}(7.3 \mathrm{meV})$ are obtained for $j_{a a}, j_{d d}$, and $j_{a d}$, respectively. The corresponding energies for YIG are $-65 \mathrm{~K}$ $(5.6 \mathrm{meV}),-30 \mathrm{~K}(2.6 \mathrm{meV})$, and $97 \mathrm{~K}(8.4 \mathrm{meV}) .{ }^{47} \mathrm{Glo}-$ bally, the relative values for BIG are in the same order of magnitude compared to YIG. In BIG films, increasing the lattice parameter [Fig. 7(a)] appears to weaken the $j_{a d}$ interaction and strengthen the $j_{a a}$ and $j_{d d}$ interactions, whereas increasing $\delta_{\mathrm{Bi}}\left[\mathrm{Fig}\right.$. 7(b)] strengthens the $j_{a d}$ interaction and weakens the other intersublattice interactions.

The Curie temperature and exchange integrals are strongly influenced by the geometry of the $[\mathrm{Fe}]-\mathrm{O}-(\mathrm{Fe})$ unit where $[\mathrm{Fe}]$ is an iron ion in octahedral site, and $(\mathrm{Fe})$ is an iron ion in tetrahedral site. Therefore, it is interesting to examine the literature data concerning the influence of a cell parameter variation on the local iron-oxygen geometry in iron garnets. Iron garnets are usually indexed in the cubic $I a-3 d$ space group ${ }^{48}$ with iron and rare-earth ions on fixed special positions. Therefore, the $[\mathrm{Fe}]-\mathrm{O}-(\mathrm{Fe})$ angle is uniquely determined by the fractional coordinates of the oxygen ion, while the $[\mathrm{Fe}]-\mathrm{O}$ and $(\mathrm{Fe})-\mathrm{O}$ distances can be expressed as a function of the cell parameter and the oxygen coordinates. As a result, an increase of the cell parameter is linked to variations of the $[\mathrm{Fe}]-\mathrm{O}-(\mathrm{Fe})$ angle and/or $[\mathrm{Fe}]-\mathrm{O}$ and $(\mathrm{Fe})-\mathrm{O}$ distances. Crystallographic data with refined oxygen parameters are required to discuss this issue. Data for $R_{3} \mathrm{Fe}_{5} \mathrm{O}_{12}(R=\mathrm{Yb}, \mathrm{Tm}, \mathrm{Er}, \mathrm{Ho}, \mathrm{Dy}, \mathrm{Y}, \mathrm{Tb}, \mathrm{Gd}$, and $\mathrm{Eu})$ can be found in the powder diffraction file (PDF)-4 crystallographic database $^{49}$ and in Ref. 50. The increase of $T_{C}$ when the rareearth radius and the cell parameter increase ${ }^{48}$ seems to be linked to a small increase of the $[\mathrm{Fe}]-\mathrm{O}-(\mathrm{Fe})$ angle without significant variation of the $[\mathrm{Fe}]-\mathrm{O}$ and $(\mathrm{Fe})-\mathrm{O}$ distances. 

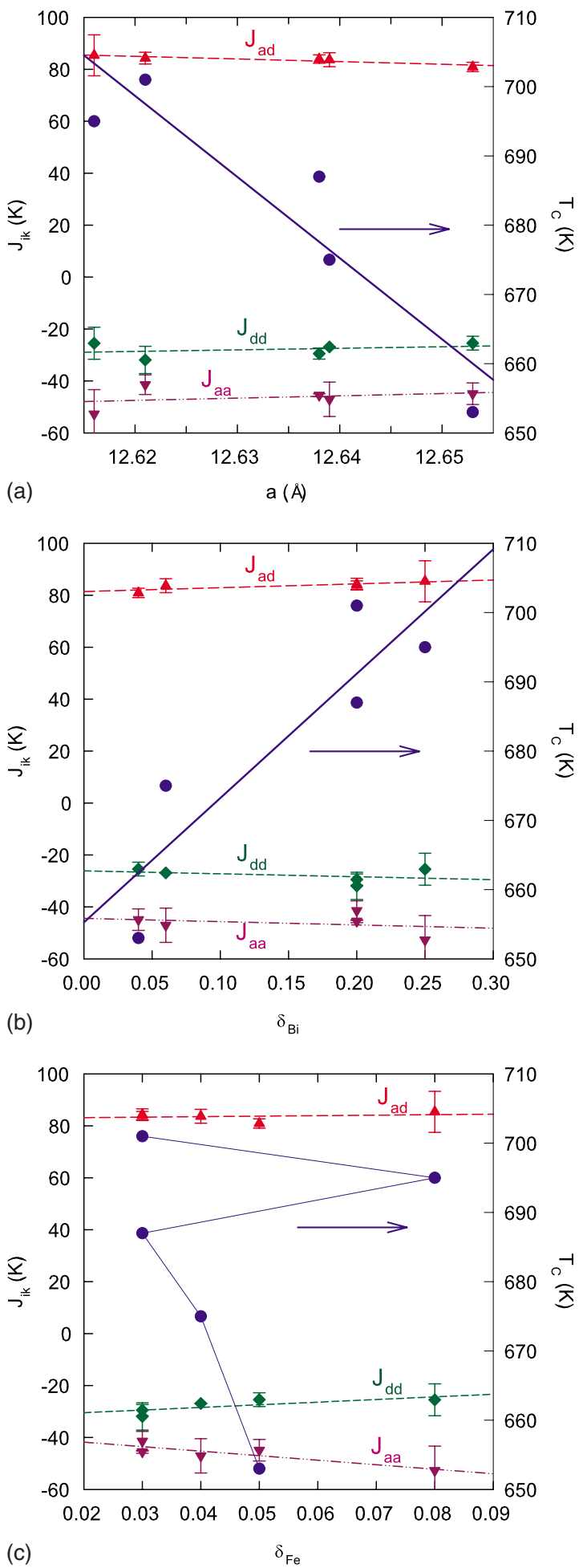

FIG. 7. (Color online) The numerically determined exchange integrals $\left(j_{a a}, j_{d d}\right.$, and $\left.j_{a d}\right)$ and the corresponding experimental $\mathrm{Cu}$ rie temperature of the BIG films as a function of (a) the lattice parameter, (b) the bismuth deficit $\delta_{\mathrm{Bi}}$, and (c) the iron deficit $\delta_{\mathrm{Fe}}$.

However, these variations are too small [e.g., [Fe]-O-(Fe) angle varies between $125^{\circ}$ and $127^{\circ}$ ] to draw firm conclusions. Indeed Geller and Colville ${ }^{51}$ reached opposite conclusions when characterizing an $\mathrm{Y}_{1.12} \mathrm{Bi}_{1.88} \mathrm{Fe}_{5} \mathrm{O}_{12}$ single crystal. They observed that the increase in cell parameter (due to bismuth substitution in YIG) seems to be linked to a decrease in the $(\mathrm{Fe})-\mathrm{O}$ distance and an increase in the $[\mathrm{Fe}]-\mathrm{O}$ distance without significant variation of the sum of the $[\mathrm{Fe}]-\mathrm{O}$ and $(\mathrm{Fe})-\mathrm{O}$ distances and without variation of the $[\mathrm{Fe}]-\mathrm{O}-(\mathrm{Fe})$ angle. But these variations are very small and the cationic mixture on the dodecahedral site implies that only average values are obtained. Therefore, it would be highly interesting to have reliable crystallographic data on $\mathrm{Bi}_{3} \mathrm{Fe}_{5} \mathrm{O}_{12}$, but this is made difficult by the fact that this compound exists only as a film. Toraya et al. ${ }^{37}$ have attempted a Rietveld refinement of x-ray diffraction data of a polycrystalline BIG film deposited on a garnet buffer layer. With respect to YIG, they found a large decrease in the $(\mathrm{Fe})-\mathrm{O}$ distance and a large increase in the $[\mathrm{Fe}]-\mathrm{O}$ distance without significant variation of the $[\mathrm{Fe}]$ $\mathrm{O}-(\mathrm{Fe})$ angle. However, the $(\mathrm{Fe})-\mathrm{O}$ distance $(\sim 1.79 \AA)$ seems unrealistically small for a $\left(\mathrm{Fe}^{3+}\right)-\mathrm{O}$ distance (usually $\sim 1.87 \AA$ ), and the $\mathrm{O}-(\mathrm{Fe})-\mathrm{O}$ angles suggest an unusually large distortion of the $\mathrm{FeO}_{4}$ tetrahedron. On the other hand, a geometry optimization of the oxygen position in BIG has recently been calculated by Oikawa et al. ${ }^{19}$ and predicts an increase of the $[\mathrm{Fe}]-\mathrm{O}-(\mathrm{Fe})$ angle of $\sim 4^{\circ}$ without significant variation of the $[\mathrm{Fe}]-\mathrm{O}$ and $(\mathrm{Fe})-\mathrm{O}$ distances when $\mathrm{BIG}$ is compared to YIG. The same authors ${ }^{19}$ obtained similar results when they calculated the oxygen coordinates using the empirical relation established by Hawthorne ${ }^{52}$ through multiple-regression analysis of structural data taken from more than 40 garnet compositions. It is of interest to note that the $\mathrm{O}-(\mathrm{Fe})-\mathrm{O}$ and $\mathrm{O}-[\mathrm{Fe}]-\mathrm{O}$ angles predicted by Oikawa et al. ${ }^{19}$ correspond to significantly smaller distortions of the octahedra and tetrahedra than in the $R_{3} \mathrm{Fe}_{5} \mathrm{O}_{12}$ compounds discussed above $\left(R=\mathrm{Yb}\right.$-Eu). ${ }^{49,50}$

The above discussion suggests that an increase in the [Fe]-O- $(\mathrm{Fe})$ angle might be involved in the $T_{C}$ increase observed in stoichiometric iron garnets when the cell parameter increases. This interpretation is consistent with the angular variation of the superexchange integral in ferrimagnetic iron garnets. ${ }^{53}$ However, our discussion only takes into account geometrical effects and considers cation- and oxygenstoichiometric compounds. The results in Fig. 5 for the series of BIG films clearly show that nonstoichiometry (both in oxygen and metal ion content) affects strongly the Curie temperature. While "stoichiometric" BIG films $D$ and $E$ reasonably agree with the linear extrapolations derived from results for Bi-substituted $\mathrm{YIG}^{4{ }^{4}}$ Bi-deficient BIG films display inverse dependencies of $T_{C}$ on lattice parameter $a$ and bismuth concentration $x_{\mathrm{Bi}}$, i.e., $T_{C}$ tends to increase when $a$ or $x_{\mathrm{Bi}}$ decreases.

Indeed cationic or oxygen vacancies are expected to modify effectively the [Fe]-O-(Fe) geometrical environment and superexchange integral. Considering the large number of parameters involved (angles, distances, spin-orbit coupling, iron oxidation state, etc.), it is difficult to identify the physical mechanism without theoretical simulations of nonstoichiometric garnet models. Considering the analysis of the magnetization data (see above), the observed trends for $j_{a d}$, $j_{a a}$, and $j_{d d}$ suggest that in off-stoichiometric BIG, a decrease in the lattice parameter or an increasing bismuth deficit results in changes in the local $[\mathrm{Fe}]-\mathrm{O}-(\mathrm{Fe})$ geometry such that the antiferromagnetic exchange between octahedral and tetrahedral iron ions is favored, thereby, enhancing the Curie temperature. 


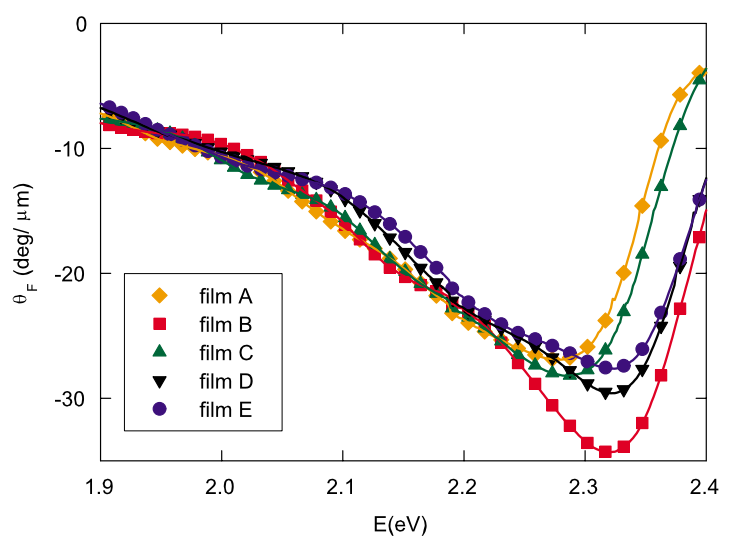

FIG. 8. (Color online) Room-temperature spectral variation of the Faraday rotation peak centered around $\sim 2.3 \mathrm{eV}$.

It is worth pointing out that the highest $T_{C}$ is observed for film $A$ characterized by comparatively large Bi deficiency, small cell parameter, and small magnetization. Since this film was grown in an oxygen-rich atmosphere and is probably not oxygen-deficient, electroneutrality suggests that $\mathrm{Fe}^{4+}$ ions might be present. This would be consistent with the small cell parameter because $\mathrm{Fe}^{4+}$ is smaller than $\mathrm{Fe}^{3+} .54,55$ Also because of its size, $\mathrm{Fe}^{4+}$ is expected to be located on the tetrahedral site, which is consistent with the small value of saturation magnetization. A small $(\mathrm{Fe})-\mathrm{O}$ distance would enhance the overlap integral and thereby the exchange integral and Curie temperature. This interpretation is consistent with a previous hydrostatic pressure study of YIG. ${ }^{56}$ In this study, it has been demonstrated that the Curie temperature of YIG increases linearly with increasing hydrostatic pressure. When linked to the variation of $T_{C}$ with decreasing lattice parameter, one can obtain a quantitative estimate of the change to $T_{C}$ for BIG. For YIG, a variation of $T_{C}$ with changes in lattice parameter, $T_{C}=-1.25 \times 10^{4} \times \Delta a$, has been determined by Podsekin et al. ${ }^{57}$ Using the experimentally observed decrease in the lattice parameter for BIG of $\Delta a$ $\approx 0.002 \mathrm{~nm}$ (Table I), an increase of $\Delta T_{C} \approx 25 \mathrm{~K}$ is obtained. This increase perfectly accounts for the experimentally observed increase in $T_{C}$ (see Table I).

\section{Room-temperature magneto-optical properties}

The Faraday rotation $\left(\theta_{F}\right)$ and ellipticity $\left(\varepsilon_{F}\right)$ of the films were measured as functions of magnetic field at different photon energies. A value of $-25^{\circ} / \mu \mathrm{m}$ for the specific Faraday rotation was observed at a photon energy of $2.25 \mathrm{eV}$ confirming literature results. ${ }^{25,29,33}$ The largest specific Faraday rotation $\left(\sim 34^{\circ} / \mu \mathrm{m}\right)$ was observed for an energy of 2.76 $\mathrm{eV}$. The measured spectral variation agrees well with the results of Chern et al. ${ }^{21}$ for $\mathrm{Y}_{3-x} \mathrm{Bi}_{x} \mathrm{Fe}_{5} \mathrm{O}_{12}(0.5 \leq x \leq 3.0)$ films deposited by PLD.

A second magneto-optical setup based on a rotating compensator method was used to measure the spectral variation of the principal Faraday rotation peak (centered at $\sim 2.3 \mathrm{eV}$ ). Figure 8 shows the specific Faraday rotation for all films in the spectral range of 1.9 to $2.4 \mathrm{eV}$. Films $B, D$, and $E$ exhibit the same spectral variation with a pronounced peak at $\sim 2.32 \mathrm{eV}$, whereas this peak occurs at lower energies of $\sim 2.27 \mathrm{eV}$ for films $A$ and $C$. It must be pointed out that films $A$ and $C$ are somewhat thicker than films $B, D$, and $E$. A qualitatively similar red shift was observed by Kahl et $a l .{ }^{25,26}$ when increasing the thickness of BIG films grown in otherwise identical conditions. These authors could not identify the cause of this apparent thickness dependence of the red shift, although a possible correlation with the x-ray coherence length was detected by x-ray diffraction. Besides, in the present case, the observed shift is somewhat larger (by $\sim 35 \%$ ) than predicted by the equation extrapolated by Kahl et $a .^{26}\left\{\Delta E(\mathrm{eV}) \sim-7.93 \times 10^{-5}(\mathrm{eV} / \mathrm{nm}) \times \Delta d(\mathrm{~nm})\right\}$. We discuss now these observations in relation to the different target compositions and oxygen pressures used for the film growth. We have seen above that variations of these experimental parameters result in variations of the stoichiometry, the lattice parameter, and the physical properties, such as the Curie temperature and the three exchange integrals $j_{a a}, j_{d d}$, and $j_{a d}$. Such changes should also result in modifications of the spectroscopic magneto-optical response of the different films. The compositional RBS analysis (see Table I) shows that both films $D$ and $E$ possess a $\mathrm{Bi} / \mathrm{Fe}$ ratio of 0.6 , whereas the other three films $(A, B, C)$ have $\mathrm{Bi} / \mathrm{Fe}$ ratio of $\sim 0.5$. Film $B$ is a particular case because it displays a spectral variation identical to that observed in the "stoichiometric" films $D$ and $E$ while presenting a significant deviation from the ideal $\mathrm{Bi} / \mathrm{Fe}$ ratio. A more detailed analysis reveals that this film not only shows an enhanced deficiency in Bi content but also an enhanced deficiency in Fe content. In the literature, the spectral variation of the magneto-optical Faraday rotation and ellipticity (or more precisely the off-diagonal permittivity tensor elements) in Bi-substituted iron garnets has been subject to intensive analysis both in terms of molecular-orbital analysis $^{10-13,15,18,19}$ and charge-transfer transitions. ${ }^{11,14,16,17,19}$ These descriptions are based on the identification of the underlying electronic transitions either by a first-principles approach $^{16,19}$ or in terms of phenomenological diamagnetic or paramagnetic transition lines. ${ }^{10-13,15,18}$ Following the frequency dispersion analysis presented by Helseth et al. ${ }^{13}$ and applied by Kahl et al., ${ }^{18}$ it turns out that within the experimentally accessible spectral range of 1.7 to $3.5 \mathrm{eV}$, only two "diamagnetic transition lines" are relevant. 13,16,18 According to Zenkov et al., ${ }^{16}$ for weakly Bi-substituted YIG the most intense transition lines correspond to a $\mathrm{FeO}_{6}$ octahedral (2.78 $\mathrm{eV})$ and $\mathrm{a}_{\mathrm{FeO}}$ tetrahedral $(3.4 \mathrm{eV}) \mathrm{O} 2 p-\mathrm{Fe}^{3+}$ chargetransfer transition. Hence, one can try to link the observed "red shift" of the rotation maximum to changes in the respective oscillator strengths $(f)$ and the densities of absorbing sites $(N)$. In this model one diamagnetic transition line is related to the octahedral $\mathrm{Fe}-\mathrm{O}$ polyhedron, whereas the second one is related to the tetrahedral $\mathrm{Fe}-\mathrm{O}$ polyhedron. In this frame, the observed "red shift" of the Faraday rotation maximum due to an increased Bismuth deficit can be identified with a reduction in either $N$ or $f$ of the tetrahedral $\mathrm{Fe}-\mathrm{O}$ polyhedra. As a consequence and in order to describe the experimental situation coherently, it is necessary to assume that an increased iron deficiency as observed in film $B$ affects essentially the octahedral $\mathrm{Fe}-\mathrm{O}$ polyhedra by decreasing either $N$ or $f$ and results in a blue shift of the Faraday rotation maximum. 
Interestingly, it is possible to use these ideas, especially the link between red shift and Bi deficiency, to suggest a possible explanation for the results of Kahl et al. ${ }^{26}$ as described above. If one accepts the fact that the $\mathrm{Bi} / \mathrm{Fe}$ ratio in PLD-grown films is larger at the film-substrate interface (as observed by us, Ref. 28, and Ref. 39), it means that increasing the film thickness would tend to increase the Bi deficiency resulting thereby in a red shift. Since Kahl et al. ${ }^{26}$ used the same target composition for all their films, it is logical that the observed red shift in their case is smaller than when we used targets with different stoichiometries.

\section{CONCLUSION}

We have studied the influence of the stoichiometry on the structural, magnetic, and magneto-optical properties of bismuth iron garnet $\left(\mathrm{Bi}_{3} \mathrm{Fe}_{5} \mathrm{O}_{12}\right)$ thin films grown by pulsed laser deposition. Films with different stoichiometries have been obtained by varying the $\mathrm{Bi} / \mathrm{Fe}$ ratio of the target and the oxygen pressure during deposition. Stoichiometry variations influence the Curie temperature $T_{C}$ by tuning the (Fe)-O-[Fe] geometry: $T_{C}$ increases when the lattice parameter decreases, contrary to what happens in the case of stoichiometric rare- earth iron garnets. The thermal variation of the magnetization, the Faraday rotation, and the Faraday ellipticity have been analyzed in the frame of the Néel two-sublattice magnetization model giving energies of $-48 \mathrm{~K}(4.1 \mathrm{meV})$, $-29 \mathrm{~K}(2.5 \mathrm{meV})$, and $84 \mathrm{~K}(7.3 \mathrm{meV})$ for the three magnetic exchange integrals $j_{a a}, j_{d d}$, and $j_{a d}$, respectively. Magneto-optical spectroscopy linked to compositional analysis by RBS shows that Bi and/or Fe deficiencies also affect the spectral variation (between 1.77 and $3.1 \mathrm{eV}$ ). Our results suggest that bismuth deficiency has an effect on the magneto-optical response of the tetrahedral $\mathrm{Fe}$ sublattice, whereas small iron deficiencies affect predominantly the magneto-optical response of the octahedral sublattice.

\section{ACKNOWLEDGMENTS}

The authors thank M. Bibes (IEF-CNRS, Orsay, France) for the high-resolution XRD experiments. B.V. thanks FNRS, CGRI, the Royal Military Academy, and the University of Liege (Belgium) for financial support. N.K. acknowledges financial support in the frame of the CNRS/CGRIFNRS cooperation Project No. 18224. T.J.J. and J.S.A. thank the UK Engineering and Physical Sciences Research Council for financial support.
*Corresponding author; b.vertruyen@ulg.ac.be

${ }^{1}$ A. K. Zvezdin and V. A. Kotov, Modern Magnetooptics and Magnetooptical Materials (Taylor \& Francis, London, 1997).

${ }^{2}$ C. Holthaus, A. Trifonov, H. Dötsch, and J. Schützmann, NDT \& E Int. 38, 129 (2005).

${ }^{3}$ M. Huang and S. Zhang, J. Mater. Res. 15, 1665 (2000).

${ }^{4}$ K. Kawano, R. A. Chakalov, G. Kong, J. S. Abell, S. Kahl, and A. M. Grishin, Physica C 372-376, 696 (2002).

${ }^{5} \mathrm{Ph}$. Vanderbemden, Z. Hong, T. A. Coombs, S. Denis, M. Ausloos, J. Schwartz, I. B. Rutel, N. H. Babu, D. A. Cardwell, and A. M. Campbell, Phys. Rev. B 75, 174515 (2007).

${ }^{6}$ M. Laulajainen, P. Paturi, J. Raittila, H. Huhtinen, A. B. Abrahamson, N. H. Anderson, and R. Laiho, J. Magn. Magn. Mater. 279, 218 (2004).

${ }^{7}$ M. Inoue and T. Fuji, J. Appl. Phys. 81, 5659 (1997).

${ }^{8}$ M. Inoue, K. Arai, T. Fuji, and M. Abe, J. Appl. Phys. 85, 5768 (1999).

${ }^{9}$ S. I. Khartsev and A. M. Grishin, Appl. Phys. Lett. 87, 122504 (2005).

${ }^{10}$ J. Yang, Y. Xu, F. Zhang, and M. Guillot, J. Phys.: Condens. Matter 18, 9287 (2006).

${ }^{11}$ G. F. Dionne and G. A. Allen, J. Appl. Phys. 73, 6127 (1993).

${ }^{12}$ G. F. Dionne and G. A. Allen, J. Appl. Phys. 75, 6372 (1994).

${ }^{13}$ L. E. Helseth, R. W. Hansen, E. I. Il'yashenko, M. Baziljevich, and T. H. Johansen, Phys. Rev. B 64, 174406 (2001).

${ }^{14}$ L. E. Helseth, J. Phys.: Condens. Matter 15, 2227 (2003).

${ }^{15}$ G. F. Dionne and G. A. Allen, J. Appl. Phys. 95, 7333 (2004).

${ }^{16}$ A. V. Zenkov and A. S. Moskvin, J. Phys.: Condens. Matter 14, 6957 (2002).

${ }^{17}$ A. V. Zenkov and A. S. Moskvin, J. Phys.: Condens. Matter 15, 2229 (2003).
${ }^{18}$ S. Kahl, V. Popov, and A. M. Grishin, J. Appl. Phys. 94, 5688 (2003); 96, 1767 (2004).

${ }^{19}$ T. Oikawa, S. Suzuki, and K. Nakao, J. Phys. Soc. Jpn. 74, 401 (2005).

${ }^{20}$ M. Y. Chern and J. S. Liaw, Jpn. J. Appl. Phys., Part 1 36, 1049 (1997).

${ }^{21}$ M. Y. Chern, F. Y. Lo, D. R. Liu, K. Yang, and J. S. Liaw, Jpn. J. Appl. Phys., Part 1 38, 6687 (1999).

${ }^{22}$ N. Adachi, V. P. Denysenkov, S. I. Khartsev, A. M. Grishin, and T. Okuda, J. Appl. Phys. 88, 2734 (2000).

${ }^{23}$ A. Jalali-Roudsar, V. P. Denysenkov, S. I. Khartsev, A. M. Grishin, N. Adachi, and T. Okuda, IEEE Trans. Magn. 37, 2454 (2001).

${ }^{24}$ S. Kahl, S. I. Khartsev, A. M. Grishin, K. Kawano, G. Kong, R. A. Chakalov, and J. S. Abell, J. Appl. Phys. 91, 9556 (2002).

${ }^{25}$ S. Kahl and A. M. Grishin, J. Appl. Phys. 93, 6945 (2003).

${ }^{26}$ S. Kahl and A. M. Grishin, J. Magn. Magn. Mater. 278, 244 (2004).

${ }^{27}$ B. M. Simion, R. Ramesh, V. G. Keramidas, G. Thomas, E. Marinero, and R. L. Pfeffer, J. Appl. Phys. 76, 6287 (1994).

${ }^{28}$ T. Tepper and C. A. Ross, J. Cryst. Growth 255, 324 (2003).

${ }^{29}$ T. Okuda, T. Katayama, T. Oikawa, H. Yamamoto, and N. Koshizuka, in Recent Advances in Magnetism and Magnetic Materials, edited by H. L. Huang and P. C. Kuo, (World Scientific, Singapore, 1989).

${ }^{30}$ T. Okuda, T. Katayama, H. Kobayashi, and N. Kobayashi, J. Appl. Phys. 67, 4944 (1990).

${ }^{31}$ N. Adachi, T. Okuda, V. P. Denysenkov, A. Jalali-Roudsar, and A. M. Grishin, J. Magn. Magn. Mater. 242-245, 775 (2002).

${ }^{32}$ P. Johansson, S. I. Khartsev, and A. M. Grishin, Thin Solid Films 515, 477 (2006). 
${ }^{33}$ T. Okuda, T. Katayama, K. Satoh, and H. Yamamoto, J. Appl. Phys. 69, 4580 (1991).

${ }^{34}$ M. Okada, S. Katayama, and K. Tominaga, J. Appl. Phys. 69, 3566 (1991).

${ }^{35}$ A. T. Thavendrarajah, M. Parvadi-Horvath, and P. E. Wigen, IEEE Trans. Magn. 25, 4015 (1989).

${ }^{36}$ S. Mino, M. Matsuoka, A. Tate, A. Shibukawa, and K. Ono, Jpn. J. Appl. Phys., Part 1 31, 1786 (1992).

${ }^{37}$ H. Toraya and T. Okuda, J. Phys. Chem. Solids 56, 1317 (1995).

${ }^{38}$ Y. Dumont, N. Keller, E. Popova, D. S. Schmool, M. Tessier, S. Bhattacharya, B. Stahl, R. M. C. Da Silva, and M. Guyot, Phys. Rev. B 76, 104413 (2007).

${ }^{39}$ R. Lux, A. Heinrich, S. Leitenmeier, T. Körner, M. Herbort, and B. Stritzker, J. Appl. Phys. 100, 113511 (2006).

${ }^{40}$ N. Keller, J. Mistrik, S. Visnovsky, D. S. Schmool, Y. Dumont, P. Renaudin, M. Guyot, and R. Krishnan, Eur. Phys. J. B 21, 67 (2001).

${ }^{41}$ P. A. Tempest, J. Appl. Crystallogr. 10, 238 (1977).

${ }^{42}$ J. P. Krumme, A. F. Otterloo, P. C. Zalm, and J. Pertruzzello, J. Appl. Phys. 64, 3965 (1988).

${ }^{43}$ S. Wittekoek, T. J. A. Popma, J. M. Robertson, and P. F. Bongers, Phys. Rev. B 12, 2777 (1975).

${ }^{44}$ P. Hansen, K. Witter, and W. Tolksdorf, Phys. Rev. B 27, 6608 (1983).
${ }^{45}$ P. Hansen, K. Witter, and W. Tolksdorf, J. Appl. Phys. 55, 1052 (1984).

${ }^{46}$ P. Hansen and J. P. Krumme, Thin Solid Films 114, 69 (1984).

${ }^{47}$ G. Dionne, J. Appl. Phys. 41, 4874 (1970).

${ }^{48}$ G. Winkler, Magnetic Garnets (Vieweg, Braunschweig, 1981).

${ }^{49}$ Powder Diffraction File PDF-4 Database, International Centre for Diffraction Data, PDF Card No. 04-009-8391 (YIG), PDF Card No. 04-009-5650 (TbIG), PDF Card No. 04-007-5211 (GdIG), PDF Card No. 04-006-1590 (EuIG).

${ }^{50}$ F. Tcheou, F. Huess, and E. F. Bertaut, Solid State Commun. 8, 1745 (1970).

${ }^{51}$ S. Geller and A. A. Colville, AIP Conference Proceedings No. 24 (AIP, New York, 1975), p. 372.

${ }^{52}$ F. C. Hawthorne, J. Solid State Chem. 37, 157 (1981).

${ }^{53}$ M. E. Lines, Phys. Rev. B 20, 3729 (1979).

${ }^{54}$ R. D. Shannon, Acta Crystallogr., Sect. A: Cryst. Phys., Diffr., Theor. Gen. Crystallogr. 32, 751 (1976).

${ }^{55}$ W. H. de Roode and C. A. P. W. van de Pavert, J. Appl. Phys. 55, 3115 (1984).

${ }^{56}$ D. Bloch, J. Phys. Chem. Solids 27, 881 (1966); G. Bocquillon, C. Loriers-Susse, and J. Loriers, High Temp. - High Press. 5, 161 (1973).

${ }^{57}$ K. Podsekin and V. N. Zaitsev, Sov. Phys. Solid State 23, 160 (1981). 